\title{
Chondrocalcinose du genou
}

\section{Calcium Pyrophosphate Deposition Disease of Knee}

\section{Tourdias • G. Barbero}

Reçu le 23 janvier 2015 ; accepté le 17 février 2015

(C) SFMU et Lavoisier SAS 2015

Un patient de 68 ans se présente aux urgences pour une gonalgie droite très invalidante évoluant depuis le matin même. L'interrogatoire retrouve une chute la veille occasionnant un traumatisme bénin du genou droit. À l'examen clinique, on retrouve des signes inflammatoires locaux (chaleur, douleur, œdème) ainsi que la présence d'un épanchement intra-articulaire. Les mouvements sont limités, aussi bien en passif qu'en actif, et il n'existe aucune laxité aux différents tests ligamentaires. Les radiographies standard objectivent des calcifications en regard des ménisques et des cartilages articulaires des deux compartiments fémorotibiaux évocatrices d'une chondrocalcinose articulaire (Fig. 1). Une ponction articulaire a ensuite été pratiquée permettant l'évacuation de $40 \mathrm{cc}$ de liquide synovial légèrement trouble dont l'étude a confirmé la présence de dépôts de cristaux de pyrophosphate de calcium au sein d'un liquide inflammatoire aseptique.

La chondrocalcinose articulaire est une arthropathie microcristalline habituellement asymptomatique. Néanmoins, cette affection rhumatismale représente la cause la plus fréquente d'arthrite aiguë chez la personne âgée [1]. Le genou est, avec le poignet, l'articulation préférentiellement atteinte, surtout lors d'une monoarthrite aiguë. Parfois, un facteur déclenchant est à l'origine d'un accès inflammatoire : affection médicochirurgicale aiguë, traumatisme, injection intra-articulaire... Le diagnostic repose sur la mise en évidence de microcristaux typiques dans le liquide synovial ou sur la présence d'images radiologiques d'incrustations calciques au niveau des fibrocartilages (ménisques, ligament triangulaire du carpe) et/ou du cartilage hyalin [1]. Le traitement de l'accès aigu est symptomatique (glace, repos articulaire et prescription prudente d'AINS) associé à une ponction articulaire évacuatrice voire une infiltration de corticoïdes [1]. Même si l'association à une

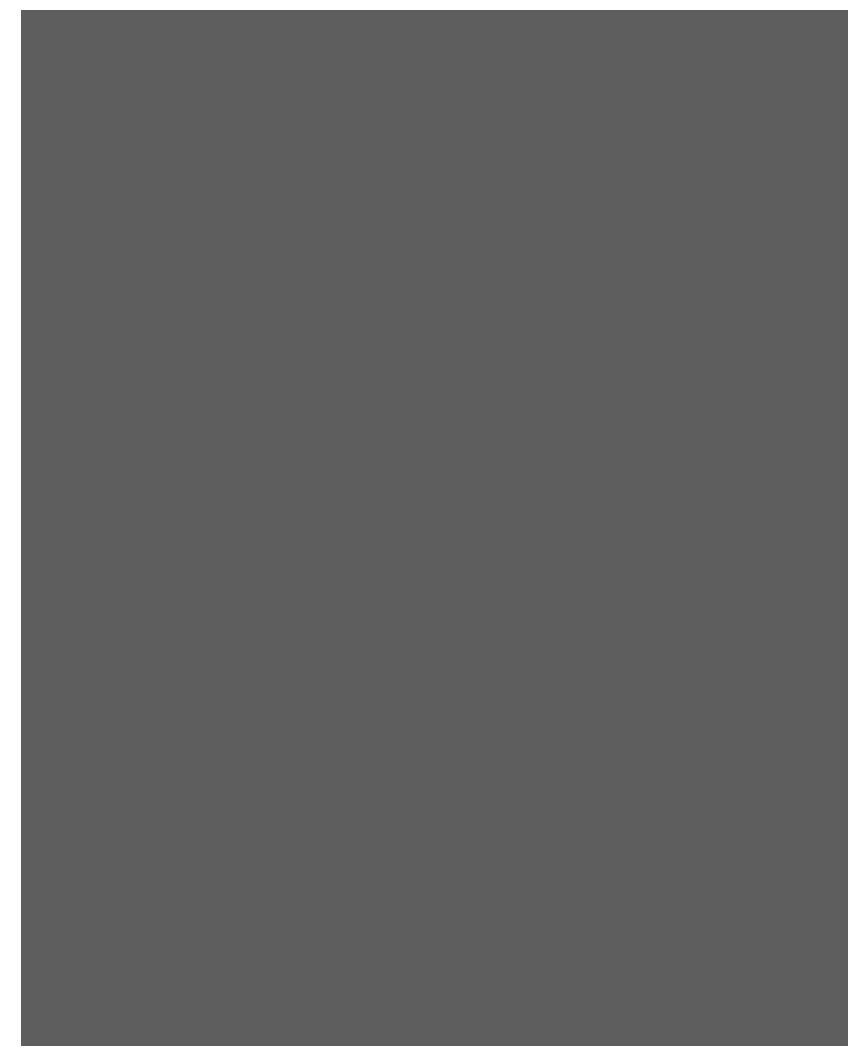

arthrite infectieuse est rare, il est préférable de demander un examen bactériologique si une ponction est réalisée.

\section{Référence}

1. Richette P, Bardin T (2011) Chondrocalcinose. Rev Prat 40:856-64

D. Tourdias $(\bowtie) \cdot$ G. Barbero

Service d'accueil des urgences - SMUR - UHCD,

centre hospitalier sud Gironde, rue Paul Langevin,

F-33210 Langon

e-mail : tourdiasdamien@yahoo.fr 